OPEN ACCESS

Edited by:

Karri P. Lamsa,

University of Szeged, Hungary

Reviewed by:

Sungmo Park,

Hospital for Sick Children, Canada

Jason D. Shepherd,

University of Utah, United States

*Correspondence: Andrei Rozov andrei.rozov@physiologie.uniheidelberg.de

Received: 01 August 2018 Accepted: 18 September 2018 Published: 10 October 2018

Citation:

Rozov A, Zakharova Y, Vazetdinova $A$ and Valiullina-Rakhmatullina $F$ (2018) The Role of Polyamine-Dependent Facilitation of Calcium Permeable AMPARs in Short-Term Synaptic

Enhancement.

Front. Cell. Neurosci. 12:345. doi: 10.3389/fncel.2018.00345

\section{The Role of Polyamine-Dependent Facilitation of Calcium Permeable AMPARs in Short-Term Synaptic Enhancement}

\author{
Andrei Rozovi,2*, Yulia Zakharova ${ }^{1}$, Alina Vazetdinova ${ }^{1}$ and \\ Fliza Valiullina-Rakhmatullina ${ }^{1}$ \\ ${ }^{1}$ Laboratory of Neurobiology, Institute of Fundamental Medicine and Biology, Kazan Federal University, Kazan, Russia, \\ ${ }^{2}$ Department of Physiology and Pathophysiology, University of Heidelberg, Heidelberg, Germany
}

Depending on subunit composition AMPA receptor channels can be subdivided into two groups: GluA2-containing calcium impermeable AMPARs, and GluA2-lacking calcium permeable, AMPARs. These two groups differ in a number of biophysical properties and, most likely, in their functional role at glutamatergic synapses. GluA2lacking channels have received a lot of attention over the last two decades mainly due to high calcium permeability, which was suggested to play a significant role in the induction of long-term synaptic plasticity in healthy tissue and neuronal death under neuropathological conditions. However, calcium permeable AMPARs possess another property that can contribute substantially to frequency dependent dynamics of synaptic efficacy. In the closed state calcium permeable AMPARs are blocked by endogenous polyamines, however, repetitive activation leads to progressive relief from the block and to the facilitation of ion flux through these channels. Polyaminedependent facilitation of AMPARs can contribute to short-term plasticity at synapses that have high initial release probability and express calcium permeable AMPARs. During synaptic transmission activity-dependent relief from polyamine block of postsynaptic calcium-permeable AMPARs either counteracts presynaptic short-term depression in a frequency-dependent manner or, under specific stimulation conditions, induces facilitation of a synaptic response. Taking into account the fact that expression of calcium permeable AMPARs is developmentally regulated, depends on network activity and increases in diseased brain states, polyamine-dependent facilitation of calcium permeable AMPARs is an important, entirely postsynaptic mechanism of synaptic gain regulation.

\section{Keywords: calcium permeable AMPA, GluA2-lacking AMPA, synapse, short-term plasticity, homeostatic plasticity, neurons, interneurons, polyamine}

\section{INTRODUCTION}

The functional properties of AMPA receptor (AMPAR) channels are, to a large extent, determined by subunit composition. Single channel conductance and calcium permeability are conferred by the presence or absence of the GluA2 subunit. In contrast to the other AMPAR subunits (GluA1, GluA3, and GluA4), the vast majority (>99\%) of GluA2 mRNA undergoes post-translational 
editing, which converts a codon for glutamine (Q), present in the GluA2 gene, to a codon for arginine (R) at position 607 in the pore forming M2 segment (Sommer et al., 1991) This alteration of charge in the channel pore, leads to a loss of calcium permeability, strongly reduces the single-channel conductance of the receptor and prevents the channel from being blocked by intracellular polyamines (Verdoorn et al., 1991). Thus, calcium permeable AMPARs (CP-AMPARs) assembled from GluA1, GluA3 and GluA4, show higher single-channel conductance and have an inwardly rectifying current-voltage relationship resulting from voltage-dependent block by intracellular polyamines. Channels containing edited GluA2 are calcium impermeable AMPARs (CI-AMPARs), which have a lower single-channel conductance and exhibit a linear voltage-current relationship (Hestrin, 1993). Over the last two decades, a great deal of attention has been paid to the functional role of the calcium permeability of CP-AMPARs (Pellegrini-Giampietro et al., 1992; Friedman et al., 1994; Gorter et al., 1997; Grooms et al., 2000; Opitz et al., 2000; Huang et al., 2002; Liu and Zukin, 2007; Lu et al., 2007; Guire et al., 2008; Wang and Gao, 2010; Rozov et al., 2012; Sanderson et al., 2016). However, this property of CP-AMPARs comes together with the voltage-dependent block by intracellular polyamines and the ability of CP-AMPARs to be relieved from this block during repetitive high frequency activation. The latter of which results in facilitation of CP-AMPAR mediated currents (Rozov et al., 1998; Rozov and Burnashev, 1999). In this mini-review we discuss the mechanism of polyamine-dependent facilitation of CP-AMPARs and its possible contribution to short-term plasticity at glutamatergic synapses.

\section{CONTRIBUTION OF CP-AMPARS TO FREQUENCY DEPENDENT FACILITATION}

\section{Mechanism of Polyamine-Dependent Facilitation of CP-AMPARs}

Under normal physiological conditions the calcium permeability of synaptic CP-AMPARs does not contribute much to postsynaptic calcium dynamics (Bollmann et al., 1998), due to the rapid inactivation of AMPAR-mediated responses and the relatively small fractional $\mathrm{Ca}^{2+}$ current $\left(\mathrm{P}_{\mathrm{f}}\right)$ through these channels. Even in recombinant systems, where the $\mathrm{Ca}^{2+}$ permeability of all expressed AMPAR channels can be ensured by transfection of vectors encoding the specific subunit, the $\mathrm{P}_{\mathrm{f}}$ of homomeric GluA1 CP-AMPARs is about $3 \%$ of the total current. For comparison, this value measured for GluN1A-GuluN2 NMDAR channels is three fold higher and exceeds 10\% (Burnashev et al., 1995). However, another feature of CP-AMPARs that comes together with high $\mathrm{Ca}^{2+}$ permeability, is their sensitivity to intracellular polyamines allows AMPAR-mediated currents to increase in an activity dependent manner.

All AMPAR channels composed of GluA1, GluA3, or/and GluA4 subunits can be blocked by endogenous polyamines such as spermidine, and spermine. Blockade of CP-AMPARs by intracellular polyamines generates inward rectification of the current-voltage (I-V) relation. However, as membrane potential becomes sufficiently positive, polyamines can permeate the AMPAR channel so that ion flux is restored resulting in a so called doubly rectifying $\mathrm{I}-\mathrm{V}$ relation (Bowie and Mayer, 1995; Burnashev et al., 1995). For a while, it was thought that polyamines act only as an open channel blocker, being more powerful at positive potentials when both the transmembrane voltage and the concentration gradients synergistically push them into the inner vestibule of the channel. Since polyamines, depending on their length, can carry up to four positive charges, the presence of these molecules in the path of ion flow greatly reduces conductance of the channel. The same line of logic was applied to explain the "weaker" polyamine block at negative potentials - a reduction of electric driving force for polyamines leads to a decrease in the probability of entering the channel in the open state (Bowie and Mayer, 1995). However, it was later shown that polyamines can enter the inner vestibule of CPAMPARs in the closed state, and the opening of the channel leads to either relief from the block or complete obstruction of the ion flow, depending on membrane potential (Rozov et al., 1998). At positive potentials polyamines are forced to permeate through the channel according to the transmembrane voltage and the concentration gradients. However, taking into account the size of these organic ions, a substantial electric driving force is needed to push polyamines through the narrow constriction of AMPARs. Even when the intracellular concentration of free polyamines is in the range of a few micromoles, a measureable outward CPAMPAR mediated current can be detected at potentials higher than $20 \mathrm{mV}$ (Donevan and Rogawski, 1995; Kamboj et al., 1995; Rozov et al., 1998). At negative potentials, opening of the CP-APMARs leads to relief from the block, since due to the transmembrane potential difference, polyamines have to leave the channel pore back to the cytosol (Figure 1A). The rate of relief strongly depends on holding potential, being faster at more negative potentials (Rozov et al., 1998). Given that release from the block is electrically driven, and the speed of re-block depends on polyamine diffusional constants, repetitive activation of the same population of CP-AMPARs results in progressive liberation of these channels from polyamine suppression and can therefore cause facilitation of AMPAR-mediated currents. Indeed, in outside-out patches expressing homomeric CP-AMPAR channels composed of GluA1, GluA4, or unedited GluA2 subunits, trains of $1 \mathrm{~ms}$ glutamate applications induced inward currents which showed robust facilitation at application frequencies of $2 \mathrm{~Hz}$ or greater. The crucial role of the polyamine block in this phenomenon was further proven by experiments where washout of polyamines lead to the loss of facilitation. Moreover, the I-V relation of the facilitated responses was almost linear, even in the presence of polyamines, pointing to activity dependent relief from the block as the main mechanism of CP-AMPAR facilitation (Rozov et al., 1998).

The apparent degree of current facilitation also dependents on the AMPARs desensitization properties. For instance, GluA1containing AMPARs, which show the slowest recovery from desensitization of all 'flip' form subunits, showed strong depression at application frequencies higher than $10 \mathrm{~Hz}$. Still, 


\section{A1

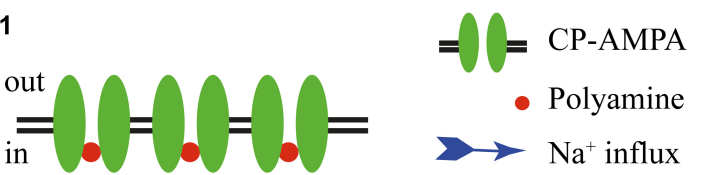

A2

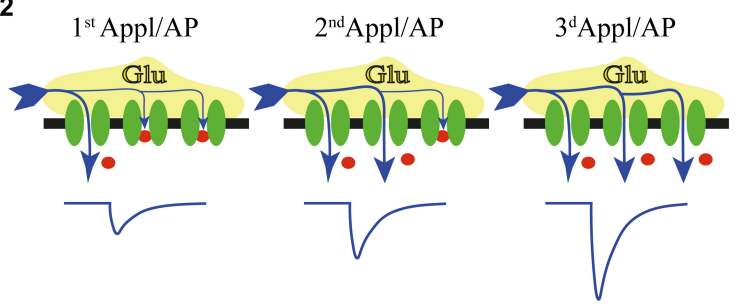

B

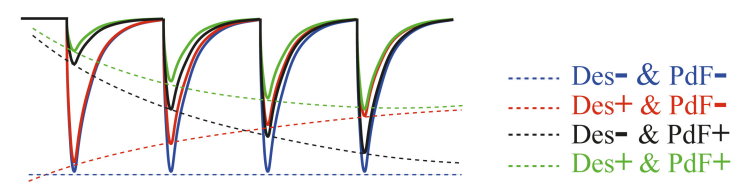

FIGURE 1 | (A) Schematic drawing of events underlying polyamine dependent facilitation of CP-AMPARs. In the closed state most of the channels are blocked by intracellular polyamines (A1). Repetitive activation of CP-AMPARs at negative potentials either by a train of brief glutamate applications or by mediator released in response to the train of action potentials, leads to progressive relief from the block and enhancement of AMPAR-mediated current amplitudes (A2). (B) Factors determining amplitudes of the currents through CP-AMPARs during high frequency activation. The blue trace shows a hypothetical response to a train of glutamate applications without contribution of channel desensitization and polyamine dependent facilitation. Experimentally this can be achieved in the presence of cyclothiazide and after washout of intracellular polyamines. Contribution of polyamine dependent facilitation (no channel desensitization) is depicted by the black trace. Note the strong impact of the polyamine block on the first response amplitude. The red trace shows gradual reduction of AMPAR-mediated currents due to channel desensitization in the absence of polyamine dependent facilitation. The green trace illustrates the product of interplay between channel desensitization and polyamine dependent facilitation of CP-AMPARs. The final amplitude dynamics in every given case will depend on: desensitization time constants, polyamine concentration and stimulation frequency.

in the presence of cyclothiazide, a positive allosteric modulator that eliminates channel desensitization, currents through GluA1containing AMPARs were facilitating in response to $33 \mathrm{~Hz}$ application trains. It is important to state that desensitization and polyamine-dependent facilitation of CP-AMPARs are two independent processes (Figure 1B). Even at high application frequencies, when the desensitization impact on response amplitudes is strong, the presence of intracellular polyamines selectively reduces the amplitude of the first CP-AMPAR mediated response and consequently diminishes the depth of the relative depression (Rozov et al., 1998).

\section{The Role of Polyamine-Dependent Facilitation of CP-AMPARs in Short-Term Synaptic Plasticity}

Polyamine-dependent facilitation can operate as an entirely postsynaptic mechanism of short-term synaptic enhancement at synapses expressing CP-AMPARs. The main requirement for significant contribution of this phenomenon to short-term plasticity is high release probability, since the same population of synaptic CP-AMPARs should be exposed to glutamate at every cycle of the presynaptic activity. Excitatory synapses onto many interneurons express CP-AMPARs and are characterized by initial high release probability. One of the most studied types of principal cell to interneuron synapses, the connection from cortical pyramidal neurons to parvalbumin positive fast spiking (FS) interneurons, provides a good model to demonstrate the involvement of polyamine-dependent facilitation in synaptic plasticity for the two following reasons. Firstly, most of the described synapses of this type have a release probability that is close to one (Reyes et al., 1998; Angulo et al., 1999; Rozov et al., 2001; Watanabe et al., 2005; Hull et al., 2009; Voinova et al., 2015). Secondly, FS cells express a high level of CP-AMPARs postsynaptically, as is the case in the vast majority of cortical interneurons (Geiger et al., 1995; Koh et al., 1995; Toth and McBain, 1998; Rozov and Burnashev, 1999). Indeed, it has been shown that in connected layer $2 / 3$ pyramidal to FS cell pairs, trains of presynaptic action potentials at stimulation frequencies of $10 \mathrm{~Hz}$ or higher trigger postsynaptic responses exhibiting prominent paired-pulse depression. However, reduction of the second EPSC amplitude relative to the first one was significantly stronger after washout of polyamines from the postsynaptic interneurons. In the presence of intracellular spermine the I$\mathrm{V}$ relation of the first EPSC was sigmoidal, while the negative limb of synaptic I-V of the second EPSC was linear, suggesting significant reduction of polyamine block. Moreover, when vesicle depletion was reduced by lowering extracellular $\mathrm{Ca}^{2+}$ concentration (from 2 to $1.5 \mathrm{mM}$ ) $10 \mathrm{~Hz}$ stimulation of excitatory inputs to layer 2/3 FS interneurons evoked EPSPs that showed paired-pulse facilitation in the cells patched with sperminecontaining intracellular solution. However, in the simultaneously recorded FS neurons dialyzed with polyamine-free pipette solution, the same train of presynaptic action potentials triggered depressing postsynaptic responses (Rozov and Burnashev, 1999).

Toth et al. (2000) provided further evidence that polyaminedependent facilitation of CP-AMPARs can serve as an entirely postsynaptic mechanism of short-term plasticity. They found that at CP-AMPA expressing synapses formed by mossy fibers onto stratum radiatum interneurons, depolarization of the postsynaptic cell from -80 to $-20 \mathrm{mV}$ leads to facilitation of the EPSCs evoked by $20 \mathrm{~Hz}$ afferent stimulation trains. Indeed, as was previously shown on recombinant CP-AMPARs, polyaminedependent facilitation measured as paired-pulse ratio (PPR) is more pronounced at less negative potentials and requires longer application trains to reach the steady-state level, since the rate of relief is voltage dependent (Rozov et al., 1998). Thus, polyamine-dependent facilitation can maintain the amplitude of the postsynaptic responses counteracting presynaptic depression at resting membrane potentials, or even switch the modality of short-term plasticity to facilitation upon depolarization of the postsynaptic neuron.

Involvement of CP-AMPARs in synaptic transmission (Rozov et al., 2012) and short-term plasticity has also been shown for connections from hippocampal CA3 to CA1 pyramidal neurons 
(Mattison et al., 2014). Mattison and colleagues showed selective expression of CP-AMPARs in the dendritic spines located on apical, but not basal, dendrites of CA1 pyramidal neurons using the following approaches: (i) Bath application of spermine causes use-dependent inhibition of excitatory responses evoked by stimulation in the stratum radiatum, but not stratum oriens; (ii) Photostimulation (microphotolysis of caged glutamate) of single dendritic spines induces AMPAR-mediated calcium influx only at apical spines; (iii) Bath application of glutamate lead to AMPAR-mediated $\mathrm{Co}^{2+}$ loading only into apical dendrites of CA1 pyramidal cells. Moreover, they found that responses triggered by photostimulation of single apical spines displayed prominent paired-pulse facilitation. The amplitudes of induced EPSCs in apical dendrites were reduced, but PPR was increased, by spermine. Finally, suppression of the recycling of GluA2containing AMPARs caused reduced apical spine responses and increased PPR. All of these observations strongly suggest both a significant impact of CP-AMPARs on synaptic efficacy and an important contribution of polyamine-dependent facilitation in short-term plasticity at stratum radiatum CA3 to CA1 pyramidal cell synapses (Mattison et al., 2014).

\section{Polyamine-Dependent Facilitation of CP-AMPARs During Development}

Expression of CP-AMPARs can be developmentally regulated. For instance, about $30 \%$ of FS interneurons in the rat prefrontal cortex express mostly calcium impermeable AMPARs during the first five postnatal weeks and in adulthood (11 weeks and older), while between these two ages ( 5 weeks to 10 weeks) $90 \%$ of FS cells of this cortical region have synaptic AMPARs that are highly sensitive to polyamines (Wang and Gao, 2010). Interestingly, in those interneurons that express predominantly CP-AMPARs, endogenous and exogenously loaded polyamines trigger synaptic facilitation of AMPA-mediated EPSCs in response to high frequency presynaptic stimulation. Contrarily, in the depressing synapses onto interneurons expressing CI-AMPARs the PPR did not depend on spermine concentration. The contribution of the developmental enhancement of CP-AMPAR expression and increase of polyamine-dependent facilitation to synaptic plasticity have also been demonstrated for local excitatory inputs to layer $2 / 3$ FS cells in the mouse visual cortex ( $\mathrm{Lu}$ et al., 2014).

Similarly to the synapses onto FS interneurons in the prefrontal cortex, at some synapses between excitatory neurons CP-AMPAR expression is developmentally regulated being higher in younger animals (Shin et al., 2005, 2007; Ho et al., 2007). At hippocampal mossy fiber to CA3 pyramidal cell connections CP-APMARs contribute significantly to the amplitude of the postsynaptic response in mice younger than P17, while in older animals the vast majority of postsynaptic channels are GluA2-containing CI-AMPARs. In slices from P10-P16 mice, evoked EPSCs have I-V relationships with clear inward rectification and application of the CP-AMPAR specific antagonist philanthotoxin-433 causes a strong reduction in their amplitudes. During this developmental stage the PPR of EPSCs evoked by $20 \mathrm{~Hz}$ trains of mossy fiber stimulation were significantly larger when postsynaptic cells were held at
$-60 \mathrm{mV}$ compared to that recorded at $+40 \mathrm{mV}$ (Ho et al., 2007). The latter observation is in good agreement with activity dependent relief from the polyamine blocks of postsynaptic CP-AMPARs. Similar transient expression of CP-AMPARs by principal neurons early in development has been shown for neocortical layer 5 pyramidal neurons (Shin et al., 2005, 2007). In P12-P14 rats, evoked AMPA-mediated EPSCs were sensitive to intracellular polyamines: (i) $\mathrm{I}-\mathrm{V}$ relationships showed robust inward rectification; (ii) washout of polyamines lead to increase of EPSC amplitudes and linearization of $\mathrm{I}-\mathrm{V}$. During the same developmental period evoked EPSCs at these synapses are characterized by profound paired-pulse facilitation that is crucially dependent on the presence of cytosolic polyamines. In older rats ( $>$ P16) synaptic AMPARs had a linear I-V relation and the amplitude of responses as well as PPRs were not affected by either polyamine washing or washout (Shin et al., 2005, 2007). Increased expression of CP-AMPARs and polyamine-dependent facilitation of AMPAR-mediated EPSCs at early developmental stages have also been shown for layer $2 / 3$ pyramidal cells and layer 4 stellate cells (Brill and Huguenard, 2008). Interestingly, synthesis of polyamines in pyramidal neurons is developmentally regulated. In young animals, the levels of spermine and its key metabolic enzyme ornithine decarboxylase are increased, and high expression of polyamines coincides in time with expression of CP-AMPARs (Shin et al., 2005), emphasizing the critical role of polyamine-dependent facilitation in brain development.

\section{FACTORS THAT CAN INFLUENCE THE CONTRIBUTION OF POLYAMINE DEPENDENT FACILITATION OF CP-AMPARS TO SHORT-TERM PLASTICITY}

\section{Regulation of CP-AMPAR and Polyamines Expression Under Physiological and Pathophysiological Conditions}

Obviously polyamine-dependent facilitation can operate only in those synapses where polyamines are expressed and CP-AMPARs significantly contribute to the postsynaptic response.

In a study published by Plant et al. (2006) it has been suggested that at Schaffer collateral to CA1 cell synapses, CP-AMPARs can be temporally recruited during LTP induction. In contrast, no involvement of CP-AMPARs has been seen in hippocampal CA1 LTP in other studies (Adesnik and Nicoll, 2007; Gray et al., 2007). However, there is a growing body of evidence in support of the first idea that functional expression of synaptic CP-AMPARs can increase temporarily in response to some LTP-inducing stimuli and/or bust of activity (Lu et al., 2007; Guire et al., 2008; Yang et al., 2010; Rozov et al., 2012; Sanderson et al., 2016). In all reported cases after LTP induction potentiated EPSCs showed either increased sensitivity to endogenous polyamines or could be partially blocked by selective CP-AMPAR antagonists. Interestingly, silencing of network activity also leads to the 
enhancement of CP-AMPARs representation at the number of synapses. This form of homeostatic plasticity has been reported in both in vitro and in vivo models (Wong-Riley and Jacobs, 2002; Bai and Wong-Riley, 2003; Thiagarajan et al., 2005; Goel et al., 2011; Soares et al., 2013; Kim and Ziff, 2014). In addition, CP-AMPARs are recruited to synapses in the amygdala during fear learning (Clem and Huganir, 2010) and in the nucleus accumbens and ventral tegmentum in models of drug addiction (Bellone et al., 2011; McCutcheon et al., 2011) CP-AMPARs have a crucial role not only in synaptic plasticity, but also in the excitotoxicity associated with several neurological disorders. In CA1 pyramidal cells ischemic insults leads to downregulation of GluA2 expression increasing the contribution of CP-AMPARs at theses synapses (PellegriniGiampietro et al., 1992; Gorter et al., 1997; Opitz et al., 2000). In hippocampal pyramidal neurons GluA2 expression can be markedly suppressed by seizures which also shifts synaptic AMPAR identity toward CP-AMPARs (Friedman et al., 1994; Grooms et al., 2000; Huang et al., 2002; Liu and Zukin, 2007).

Expression of the second key player for CP-AMPAR facilitation, polyamines, also depends on the cell type, animal age and can also be affected during pathogenesis. Although the neurons are fully equipped for polyamine synthesis and degradation, the expression of some the necessary enzymes is under strong developmental control (Bernstein and Muller, 1995). Biosynthesis of polyamines requires ornithine decarboxylase (ODC) to catalyze the formation of putrescine, which is the precursor of the other two polyamines. The biochemical and immunohistochemical studies show that in the rat brain the highest ODC activity is at P0 (Ichikawa et al., 1997). Then ODC activity rapidly declines within the first 2 postnatal weeks. In adulthood ODC immunoreactivity is strongly reduced and has region-specific and cell-type-specific expression patterns. Usage of antibodies against spermine/spermidine has confirmed different levels of polyamine synthesis in distinct neuronal populations. For instance, in the hippocampus spermine/spermidine immunolabeling of neurons located in the CA1 and CA3 regions was weaker compared to the cells in the CA2 area, and labeling of interneurons was higher relative to pyramidal cells. Similar cell-type specificity of polyamine expression was detected also in few neocortical areas, hypothalamus, nucleus ruber, dorsolateral thalamus, raphe nuclei, central and lateral amygdale, and the cerebellar cortex (Laube et al., 2002; Krauss et al., 2007).

Elevated ODC activity and putrescine concentrations have been detected in the brain in pathological conditions such as Alzheimer's disease (AD) and ischemia (Paschen et al., 1991; Bernstein and Muller, 1995; Morrison and Kish, 1995; Keinanen et al., 1997). Nilsson et al have found evidence for translocation of ODC from the nucleus to the cytoplasm of neocortical pyramidal cells at the early stages of Alzheimer's disease. Elevated expression of ODC was also found in cerebellar Purkinje cells and in the hippocampus (Nilsson et al., 2006). Similar alterations of ODC expression and polyamine production were observed during cerebral ischemia. The ODC mRNA levels increases after ischemia onset together with putrescine content, while spermidine and spermine levels remain constant or decline. Interestingly, in healthy tissue, increased ODC activity results in elevation of all three polyamines (Temiz et al., 2005).

Thus, both synaptic expression on CP-AMPARs and polyamine synthesis alternate during development and in response to physiological and pathophysiological stimuli. These alternations might have a significant impact on synaptic short-term plasticity at the affected synapses by means of polyamine-dependent facilitation. However, unfortunately this issue is often outside the scope of these types of studies.

\section{Modulation of CP-AMPAR Polyamine Sensitivity by Auxiliary Subunits}

Biophysical properties of synaptic CP-AMPARs that are important for polyamine-dependent facilitation can be drastically affected by interaction with auxiliary subunits such as transmembrane AMPAR regulatory proteins (TARPs), cornichon homologues proteins (CNIHs) and germ cell specific gene 1-like protein (GSG1L). TARPs can be subdivided on the basis of functional differences and sequence homologies into the type 1 TARPs comprising the subunits $\gamma 2, \gamma 3, \gamma 4$, and $\gamma 8$, and the type II TARPs, $\gamma 5$, and $\gamma 7$. Type 2 TARPs modulate only CI-AMPARs (Kato et al., 2008). Among type 1 TARPs $\gamma 2$ is the most studied member. Interaction of CP-AMPARs with $\gamma 2$ slows desensitization and deactivation and greatly attenuates intracellular polyamine block across all voltages, most obviously at depolarized potentials (Soto et al., 2007; Soto et al., 2014). Three other members of TARP1 family, $\gamma 3, \gamma 4$, and $\gamma 8$, regulate CP-AMPA receptors in a qualitatively similar manner (Cho et al., 2007). Interaction with CNIHs also reduce CP-AMPARs desensitization and polyamine sensitivity. Resent findings suggest that both TARPs and CNIHs relieve channel block by enhancing the rate of blocker permeation (Brown et al., 2018). Similarly, to type 1 TARPs and CNIHs, GSG1L slows deactivation and desensitization of CP-AMPARs (Shanks et al., 2012). However, in contrast to other auxiliary AMPAR subunits GSG1L increases intracellular polyamine block of these channels greatly suppressing outward currents in the presence of intracellular polyamines (McGee et al., 2015; Greger et al., 2017). Obviously, modulation of polyamine sensitivity and the desensitization properties of CP-AMPARs by the auxiliary subunits might have a strong influence on the rate, frequency dependence and magnitude of polyamine-dependent facilitation. These questions will have to be addressed in future studies.

\section{CONCLUDING REMARKS}

Thus, the polyamine dependent facilitation of CP-AMPARs can play an important role as an alternative postsynaptic mechanism of short-term synaptic enhancement: (i) in a number of excitatory synapses converging to interneurons in the developing and adult brain; (ii) at connections between glutamatergic neurons during early postnatal development. 
Further investigation into the role of polyamine dependent facilitation of CP-AMPARs at synapses where expressions of CP-AMPARs and polyamines are changed by network activity, homeostatically driven plasticity or neurological disorders, is essential for better understanding of the fuctioning of neuronal networks.

\section{AUTHOR CONTRIBUTIONS}

All authors listed have made a substantial, direct and intellectual contribution to the work, and approved it for publication.

\section{REFERENCES}

Adesnik, H., and Nicoll, R. A. (2007). Conservation of glutamate receptor 2containing AMPA receptors during long-term potentiation. J. Neurosci. 27, 4598-4602. doi: 10.1523/JNEUROSCI.0325-07.2007

Angulo, M. C., Rossier, J., and Audinat, E. (1999). Postsynaptic glutamate receptors and integrative properties of fast-spiking interneurons in the rat neocortex. J. Neurophysiol. 82, 1295-1302. doi: 10.1152/jn.1999.82.3.1295

Bai, X., and Wong-Riley, M. T. (2003). Neuronal activity regulates protein and gene expressions of GluR2 in postnatal rat visual cortical neurons in culture. J. Neurocytol. 32, 71-78. doi: 10.1023/A:1027380315902

Bellone, C., Mameli, M., and Luscher, C. (2011). In utero exposure to cocaine delays postnatal synaptic maturation of glutamatergic transmission in the VTA. Nat. Neurosci. 14, 1439-1446. doi: 10.1038/nn.2930

Bernstein, H. G., and Muller, M. (1995). Increased immunostaining for L-ornithine decarboxylase occurs in neocortical neurons of Alzheimer's disease patients. Neurosci. Lett. 186, 123-126. doi: 10.1016/0304-3940(95)11 301-C

Bollmann, J. H., Helmchen, F., Borst, J. G., and Sakmann, B. (1998). Postsynaptic $\mathrm{Ca}^{2+}$ influx mediated by three different pathways during synaptic transmission at a calyx-type synapse. J. Neurosci. 18, 10409-10419. doi: 10.1523/ JNEUROSCI.18-24-10409.1998

Bowie, D., and Mayer, M. L. (1995). Inward rectification of both AMPA and kainate subtype glutamate receptors generated by polyamine-mediated ion channel block. Neuron 15, 453-462. doi: 10.1016/0896-6273(95)90049-7

Brill, J., and Huguenard, J. R. (2008). Sequential changes in AMPA receptor targeting in the developing neocortical excitatory circuit. J. Neurosci. 28, 1391813928. doi: 10.1523/JNEUROSCI.3229-08.2008

Brown, P. M. G. E., McGuire, H., and Bowie, D. (2018). Stargazin and cornichon-3 relieve polyamine block of AMPA receptors by enhancing blocker permeation. J. Gen. Physiol. 150, 67-82. doi: 10.1085/jgp.201711895

Burnashev, N., Zhou, Z., Neher, E., and Sakmann, B. (1995). Fractional calcium currents through recombinant GluR channels of the NMDA, AMPA and kainate receptor subtypes. J. Physiol. 485(Pt 2), 403-418.

Cho, C. H., St-Gelais, F., Zhang, W., Tomita, S., and Howe, J. R. (2007). Two families of TARP isoforms that have distinct effects on the kinetic properties of AMPA receptors and synaptic currents. Neuron 55, 890-904. doi: 10.1016/j. neuron.2007.08.024

Clem, R. L., and Huganir, R. L. (2010). Calcium-permeable AMPA receptor dynamics mediate fear memory erasure. Science 330, 1108-1112. doi: 10.1126/ science. 1195298

Donevan, S. D., and Rogawski, M. A. (1995). Intracellular polyamines mediate inward rectification of $\mathrm{Ca}(2+)$-permeable alpha-amino-3-hydroxy-5-methyl4-isoxazolepropionic acid receptors. Proc. Natl. Acad. Sci. U.S.A. 92, 9298-9302. doi: $10.1073 /$ pnas. 92.20 .9298

Friedman, L. K., Pellegrini-Giampietro, D. E., Sperber, E. F., Bennett, M. V., Moshe, S. L., and Zukin, R. S. (1994). Kainate-induced status epilepticus alters glutamate and GABAA receptor gene expression in adult rat hippocampus: an in situ hybridization study. J. Neurosci. 14, 2697-2707. doi: 10.1523/ JNEUROSCI.14-05-02697.1994

Geiger, J. R., Melcher, T., Koh, D. S., Sakmann, B., Seeburg, P. H., Jonas, P., et al. (1995). Relative abundance of subunit mRNAs determines gating and $\mathrm{Ca}^{2+}$

\section{FUNDING}

Research was supported by RSBF (17-04-01528 A) and the subsidy allocated to Kazan Federal University for the state assignment in the sphere of scientific activities (6.2313.2017/4.6). The work was performed according to the Program of Competitive Growth of Kazan Federal University.

\section{ACKNOWLEDGMENTS}

We thank David Jappy for useful comments on the manuscript.

permeability of AMPA receptors in principal neurons and interneurons in rat CNS. Neuron 15, 193-204. doi: 10.1016/0896-6273(95)90076-4

Goel, A., Xu, L. W., Snyder, K. P., Song, L., Goenaga-Vazquez, Y., Megill, A., et al. (2011). Phosphorylation of AMPA receptors is required for sensory deprivation-induced homeostatic synaptic plasticity. PLoS One 6:e18264. doi: 10.1371/journal.pone.0018264

Gorter, J. A., Petrozzino, J. J., Aronica, E. M., Rosenbaum, D. M., Opitz, T., Bennett, M. V., et al. (1997). Global ischemia induces downregulation of Glur2 mRNA and increases AMPA receptor-mediated $\mathrm{Ca}^{2+}$ influx in hippocampal CA1 neurons of gerbil. J. Neurosci. 17, 6179-6188. doi: 10.1523/JNEUROSCI. 17-16-06179.1997

Gray, E. E., Fink, A. E., Sarinana, J., Vissel, B., and O’Dell, T. J. (2007). Long-term potentiation in the hippocampal CA1 region does not require insertion and activation of GluR2-lacking AMPA receptors. J. Neurophysiol. 98, 2488-2492. doi: 10.1152/jn.00473.2007

Greger, I. H., Watson, J. F., and Cull-Candy, S. G. (2017). Structural and functional architecture of AMPA-Type glutamate receptors and their auxiliary proteins. Neuron 94, 713-730. doi: 10.1016/j.neuron.2017. 04.009

Grooms, S. Y., Opitz, T., Bennett, M. V., and Zukin, R. S. (2000). Status epilepticus decreases glutamate receptor 2 mRNA and protein expression in hippocampal pyramidal cells before neuronal death. Proc. Natl. Acad. Sci. U.S.A. 97, 36313636. doi: 10.1073/pnas.97.7.3631

Guire, E. S., Oh, M. C., Soderling, T. R., and Derkach, V. A. (2008). Recruitment of calcium-permeable AMPA receptors during synaptic potentiation is regulated by CaM-kinase I. J. Neurosci. 28, 6000-6009. doi: 10.1523/JNEUROSCI.038408.2008

Hestrin, S. (1993). Different glutamate receptor channels mediate fast excitatory synaptic currents in inhibitory and excitatory cortical neurons. Neuron 11, 1083-1091. doi: 10.1016/0896-6273(93)90221-C

Ho, M. T., Pelkey, K. A., Topolnik, L., Petralia, R. S., Takamiya, K., Xia, J., et al. (2007). Developmental expression of $\mathrm{Ca} 2+$-permeable AMPA receptors underlies depolarization-induced long-term depression at mossy fiber CA3 pyramid synapses. J. Neurosci. 27, 11651-11662. doi: 10.1523/JNEUROSCI. 2671-07.2007

Huang, Y., Doherty, J. J., and Dingledine, R. (2002). Altered histone acetylation at glutamate receptor 2 and brain-derived neurotrophic factor genes is an early event triggered by status epilepticus. J. Neurosci. 22, 8422-8428. doi: 10.1523/ JNEUROSCI.22-19-08422.2002

Hull, C., Isaacson, J. S., and Scanziani, M. (2009). Postsynaptic mechanisms govern the differential excitation of cortical neurons by thalamic inputs. J. Neurosci. 29, 9127-9136. doi: 10.1523/JNEUROSCI.5971-08.2009

Ichikawa, M., Maeda, T., and Handa, J. (1997). Ornithine decarboxylase in developing brain of normal and hydrocephalic rats. Biochemical and immunohistochemical studies. Childs Nerv. Syst. 13, 578-583. doi: 10.1007/ s003810050143

Kamboj, S. K., Swanson, G. T., and Cull-Candy, S. G. (1995). Intracellular spermine confers rectification on rat calcium-permeable AMPA and kainate receptors. J. Physiol. 486(Pt 2), 297-303.

Kato, A. S., Siuda, E. R., Nisenbaum, E. S., and Bredt, D. S. (2008). AMPA receptor subunit-specific regulation by a distinct family of type II TARPs. Neuron 59, 986-996. doi: 10.1016/j.neuron.2008.07.034 
Keinanen, R., Miettinen, S., Yrjanheikki, J., and Koistinaho, J. (1997). Induction of ornithine decarboxylase mRNA in transient focal cerebral ischemia in the rat. Neurosci. Lett. 239, 69-72. doi: 10.1016/S0304-3940(97) 00890-2

Kim, S., and Ziff, E. B. (2014). Calcineurin mediates synaptic scaling via synaptic trafficking of $\mathrm{Ca}^{2+}$-permeable AMPA receptors. PLoS Biol. 12:e1001900. doi: 10.1371/journal.pbio.1001900

Koh, D. S., Burnashev, N., and Jonas, P. (1995). Block of native Ca(2+ )-permeable AMPA receptors in rat brain by intracellular polyamines generates double rectification. J. Physiol. 486(Pt 2), 305-312.

Krauss, M., Weiss, T., Langnaese, K., Richter, K., Kowski, A., Veh, R. W., et al. (2007). Cellular and subcellular rat brain spermidine synthase expression patterns suggest region-specific roles for polyamines, including cerebellar presynaptic function. J. Neurochem. 103, 679-693. doi: 10.1111/j.1471-4159.2007. 04770.x

Laube, G., Bernstein, H. G., Wolf, G., and Veh, R. W. (2002). Differential distribution of spermidine/spermine-like immunoreactivity in neurons of the adult rat brain. J. Comp. Neurol. 444, 369-386. doi: 10.1002/cne.10157

Liu, S. J., and Zukin, R. S. (2007). $\mathrm{Ca}^{2+}$-permeable AMPA receptors in synaptic plasticity and neuronal death. Trends Neurosci. 30, 126-134. doi: 10.1016/j.tins. 2007.01.006

Lu, J., Tucciarone, J., Lin, Y., and Huang, Z. J. (2014). Input-specific maturation of synaptic dynamics of parvalbumin interneurons in primary visual cortex. Proc. Natl. Acad. Sci. U.S.A. 111, 16895-16900. doi: 10.1073/pnas.1400694111

Lu, Y., Allen, M., Halt, A. R., Weisenhaus, M., Dallapiazza, R. F., Hall, D. D., et al. (2007). Age-dependent requirement of AKAP150-anchored PKA and GluR2lacking AMPA receptors in LTP. EMBO J. 26, 4879-4890. doi: 10.1038/sj.emboj. 7601884

Mattison, H. A., Bagal, A. A., Mohammadi, M., Pulimood, N. S., Reich, C. G., Alger, B. E., et al. (2014). Evidence of calcium-permeable AMPA receptors in dendritic spines of CA1 pyramidal neurons. J. Neurophysiol. 112, 263-275. doi: 10.1152/jn.00578.2013

McCutcheon, J. E., Wang, X., Tseng, K. Y., Wolf, M. E., and Marinelli, M. (2011). Calcium-permeable AMPA receptors are present in nucleus accumbens synapses after prolonged withdrawal from cocaine self-administration but not experimenter-administered cocaine. J. Neurosci. 31, 5737-5743. doi: 10.1523/ JNEUROSCI.0350-11.2011

McGee, T. P., Bats, C., Farrant, M., and Cull-Candy, S. G. (2015). Auxiliary subunit GSG1L Acts to suppress calcium-permeable AMPA receptor function. J. Neurosci. 35, 16171-16179. doi: 10.1523/JNEUROSCI.215215.2015

Morrison, L. D., and Kish, S. J. (1995). Brain polyamine levels are altered in Alzheimer's disease. Neurosci. Lett. 197, 5-8. doi: 10.1016/0304-3940(95) 11881-V

Nilsson, T., Bogdanovic, N., Volkman, I., Winblad, B., Folkesson, R., and Benedikz, E. (2006). Altered subcellular localization of ornithine decarboxylase in Alzheimer's disease brain. Biochem. Biophys. Res. Commun. 344, 640-646. doi: 10.1016/j.bbrc.2006.03.191

Opitz, T., Grooms, S. Y., Bennett, M. V., and Zukin, R. S. (2000). Remodeling of alpha-amino-3-hydroxy-5-methyl-4-isoxazole-propionic acid receptor subunit composition in hippocampal neurons after global ischemia. Proc. Natl. Acad. Sci. U.S.A. 97, 13360-13365. doi: 10.1073/pnas.97.24. 13360

Paschen, W., Csiba, L., Rohn, G., and Bereczki, D. (1991). Polyamine metabolism in transient focal ischemia of rat brain. Brain Res. 566, 354-357. doi: 10.1016/ 0006-8993(91)91726-H

Pellegrini-Giampietro, D. E., Zukin, R. S., Bennett, M. V., Cho, S., and Pulsinelli, W. A. (1992). Switch in glutamate receptor subunit gene expression in CA1 subfield of hippocampus following global ischemia in rats. Proc. Natl. Acad. Sci. U.S.A. 89, 10499-10503. doi: 10.1073/pnas.89.21. 10499

Plant, K., Pelkey, K. A., Bortolotto, Z. A., Morita, D., Terashima, A., McBain, C. J., et al. (2006). Transient incorporation of native GluR2-lacking AMPA receptors during hippocampal long-term potentiation. Nat. Neurosci. 9, 602-604. doi: $10.1038 / \mathrm{nn} 1678$

Reyes, A., Lujan, R., Rozov, A., Burnashev, N., Somogyi, P., and Sakmann, B. (1998). Target-cell-specific facilitation and depression in neocortical circuits. Nat. Neurosci. 1, 279-285. doi: 10.1038/1092
Rozov, A., and Burnashev, N. (1999). Polyamine-dependent facilitation of postsynaptic AMPA receptors counteracts paired-pulse depression. Nature 401, 594-598. doi: 10.1038/44151

Rozov, A., Burnashev, N., Sakmann, B., and Neher, E. (2001). Transmitter release modulation by intracellular $\mathrm{Ca} 2+$ buffers in facilitating and depressing nerve terminals of pyramidal cells in layer $2 / 3$ of the rat neocortex indicates a target cell-specific difference in presynaptic calcium dynamics. J. Physiol. 531, 807-826. doi: 10.1111/j.1469-7793.2001.0807h.x

Rozov, A., Zilberter, Y., Wollmuth, L. P., and Burnashev, N. (1998). Facilitation of currents through rat $\mathrm{Ca}^{2+}$-permeable AMPA receptor channels by activity-dependent relief from polyamine block. J. Physiol. 511(Pt 2), 361-377.

Rozov, A., Zivkovic, A. R., and Schwarz, M. K. (2012). Homerl gene products orchestrate $\mathrm{Ca}(2+$ )-permeable AMPA receptor distribution and LTP expression. Front. Synaptic Neurosci. 4:4. doi: 10.3389/fnsyn.2012.00004

Sanderson, J. L., Gorski, J. A., and Dell'Acqua, M. L. (2016). NMDA receptordependent LTD requires transient synaptic incorporation of $\mathrm{Ca}(2)(+)$ permeable AMPARs mediated by AKAP150-anchored PKA and Calcineurin. Neuron 89, 1000-1015. doi: 10.1016/j.neuron.2016.01.043

Shanks, N. F., Savas, J. N., Maruo, T., Cais, O., Hirao, A., Oe, S., et al. (2012) Differences in AMPA and kainate receptor interactomes facilitate identification of AMPA receptor auxiliary subunit GSG1L. Cell Rep. 1, 590-598. doi: 10.1016/ j.celrep.2012.05.004

Shin, J., Shen, F., and Huguenard, J. (2007). PKC and polyamine modulation of GluR2-deficient AMPA receptors in immature neocortical pyramidal neurons of the rat. J. Physiol. 581, 679-691. doi: 10.1113/jphysiol.2007. 130963

Shin, J., Shen, F., and Huguenard, J. R. (2005). Polyamines modulate AMPA receptor-dependent synaptic responses in immature layer v pyramidal neurons. J. Neurophysiol. 93, 2634-2643. doi: 10.1152/jn.01054.2004

Soares, C., Lee, K. F., Nassrallah, W., and Beique, J. C. (2013). Differential subcellular targeting of glutamate receptor subtypes during homeostatic synaptic plasticity. J. Neurosci. 33, 13547-13559. doi: 10.1523/JNEUROSCI. 1873-13.2013

Sommer, B., Kohler, M., Sprengel, R., and Seeburg, P. H. (1991). RNA editing in brain controls a determinant of ion flow in glutamate-gated channels. Cell 67, 11-19. doi: 10.1016/0092-8674(91)90568-J

Soto, D., Coombs, I. D., Gratacos-Batlle, E., Farrant, M., and Cull-Candy, S. G. (2014). Molecular mechanisms contributing to TARP regulation of channel conductance and polyamine block of calcium-permeable AMPA receptors. J. Neurosci. 34, 11673-11683. doi: 10.1523/JNEUROSCI.0383-14. 2014

Soto, D., Coombs, I. D., Kelly, L., Farrant, M., and Cull-Candy, S. G. (2007). Stargazin attenuates intracellular polyamine block of calcium-permeable AMPA receptors. Nat. Neurosci. 10, 1260-1267. doi: 10.1038/nn1966

Temiz, C., Dogan, A., Baskaya, M. K., and Dempsey, R. J. (2005). Effect of difluoromethylornithine on reperfusion injury after temporary middle cerebral artery occlusion. J. Clin. Neurosci. 12, 449-452. doi: 10.1016/j.jocn.2004. 05.019

Thiagarajan, T. C., Lindskog, M., and Tsien, R. W. (2005). Adaptation to synaptic inactivity in hippocampal neurons. Neuron 47, 725-737. doi: 10.1016/j.neuron. 2005.06.037

Toth, K., and McBain, C. J. (1998). Afferent-specific innervation of two distinct AMPA receptor subtypes on single hippocampal interneurons. Nat. Neurosci. 1, 572-578. doi: 10.1038/2807

Toth, K., Suares, G., Lawrence, J. J., Philips-Tansey, E., and McBain, C. J. (2000). Differential mechanisms of transmission at three types of mossy fiber synapse. J. Neurosci. 20, 8279-8289. doi: 10.1523/JNEUROSCI.20-22-08279.2000

Verdoorn, T. A., Burnashev, N., Monyer, H., Seeburg, P. H., and Sakmann, B. (1991). Structural determinants of ion flow through recombinant glutamate receptor channels. Science 252, 1715-1718. doi: 10.1126/science. 1710829

Voinova, O., Valiullina, F., Zakharova, Y., Mukhtarov, M., Draguhn, A., and Rozov, A. (2015). Layer specific development of neocortical pyramidal to fast spiking cell synapses. Front. Cell. Neurosci. 9:518. doi: 10.3389/fncel.2015. 00518

Wang, H. X., and Gao, W. J. (2010). Development of calcium-permeable AMPA receptors and their correlation with NMDA receptors in fast-spiking 
interneurons of rat prefrontal cortex. J. Physiol. 588, 2823-2838. doi: 10.1113/ jphysiol.2010.187591

Watanabe, J., Rozov, A., and Wollmuth, L. P. (2005). Target-specific regulation of synaptic amplitudes in the neocortex. J. Neurosci. 25, 1024-1033. doi: 10.1523/ JNEUROSCI.3951-04.2005

Wong-Riley, M. T., and Jacobs, P. (2002). AMPA glutamate receptor subunit 2 in normal and visually deprived macaque visual cortex. Vis. Neurosci. 19, 563-573. doi: $10.1017 /$ S0952523802195022

Yang, Y., Wang, X. B., and Zhou, Q. (2010). Perisynaptic GluR2-lacking AMPA receptors control the reversibility of synaptic and spines modifications. Proc. Natl. Acad. Sci. U.S.A. 107, 11999-12004. doi: 10.1073/pnas.0913004107
Conflict of Interest Statement: The authors declare that the research was conducted in the absence of any commercial or financial relationships that could be construed as a potential conflict of interest.

Copyright (c) 2018 Rozov, Zakharova, Vazetdinova and Valiullina-Rakhmatullina. This is an open-access article distributed under the terms of the Creative Commons Attribution License (CC BY). The use, distribution or reproduction in other forums is permitted, provided the original author(s) and the copyright owner(s) are credited and that the original publication in this journal is cited, in accordance with accepted academic practice. No use, distribution or reproduction is permitted which does not comply with these terms. 